\title{
La représentation du nonverbal dans la communication écrite médiatisée par ordinateur
}

\section{Michel Marcoccia}

\section{OpenEdition}

\section{Journals}

Édition électronique

URL : https://journals.openedition.org/communicationorganisation/2431

DOI : 10.4000/communicationorganisation.2431

ISSN : 1775-3546

\section{Éditeur}

Presses universitaires de Bordeaux

\section{Édition imprimée}

Date de publication : 1 novembre 2000

ISSN : 1168-5549

Référence électronique

Michel Marcoccia, «La représentation du nonverbal dans la communication écrite médiatisée par ordinateur », Communication et organisation [En ligne], 18 | 2000, mis en ligne le 27 mars 2012, consulté le 05 août 2021. URL : http://journals.openedition.org/communicationorganisation/2431 ; DOI : https://doi.org/10.4000/communicationorganisation.2431

Ce document a été généré automatiquement le 5 août 2021.

(C) Presses universitaires de Bordeaux 


\title{
La représentation du nonverbal dans la communication écrite médiatisée par ordinateur
}

\author{
Michel Marcoccia
}

1 La Communication Écrite Médiatisée par Ordinateur (courrier électronique, forums de discussion. Internet Relay Chat) est depuis quelques années un moyen de communication très courant au sein des organisations, et est parfois considérée comme un outil privilégié de travail collectif et coopératif. Ce mode de communication peut être qualifié de «conversation écrite » car il repose sur une utilisation du code écrit dans une dynamique conversationnelle. Toutefois, une partie des possibilités offertes par la conversation en face à face disparaissent naturellement avec la Communication Écrite Médiatisée par Ordinateur (ou CEMO) : absence de marqueurs personnels, absence de données paraverbales et non-verbales, etc. Les utilisateurs de dispositifs de CEMO compensent ces absences en «simulant» les dimensions non-verbales et paraverbales de l'oralité grâce à la représentation de ces indices par diverses conventions graphiques. En nous basant sur l'étude de trois forums de discussion francophones ${ }^{1}$, nous ferons une typologie des divers moyens par lesquels les utilisateurs représentent ces données non-verbales et paraverbales: smileys, signatures-autoportraits, ponctuation expressive, utilisation de capitales, commentaires métadiscursifs. Nous verrons que ces représentations écrites du non-verbal et du paraverbal n'ont pas seulement une valeur ludique, esthétique ou distinctive mais qu'elles recouvrent les fonctions qu'ont le non-verbal et le paraverbal dans la communication en face à face. Du même coup, ces formes de simulation du non-verbal et du paraverbal dans la CEMO peuvent être vues comme des preuves de l'importance du non-verbal pour la communication dans les organisations et dans les groupes réels ou « virtuels». 


\section{La communication écrite médiatisée par ordinateur}

2 La CEMO est une forme de communication hybride car elle relève à la fois de la communication écrite et de la conversation. Le code utilisé est l'écrit, mais les échanges de messages entrent dans une dynamique conversationnelle, essentiellement à cause de la rapidité de la rédaction et de la transmission des messages. De ce point de vue, la CEMO se distingue d'autres types d'écrits conversationnels comme les écrits épistolaires (Melançon 1996). Dans la conversation en face à face comme dans la CEMO, il y a une relation dialogale entre interventions initiative et réactive. De plus, les indices de l'engagement du locuteur dans son énonciation (pronoms, modalisations) rapprochent la CEMO de l'échange oral (Yates 1996: 40-43). Enfin, de nombreux travaux ${ }^{2}$ ont montré que le style des écrits médiatisés par ordinateur est marqué par l'oralité.

Toutefois, la CEMO se distingue de la conversation orale sur au moins trois aspects.

4 Lorsqu'on ne connaît pas préalablement l'identité des internautes avec lesquels on échange des messages, on n'a d'informations que sur leur adresse électronique. Les autres aspects de l'identité (nom, sexe, âge, etc.) peuvent correspondre à une identité fictive.

5 Dans un forum de discussion ou dans une liste de diffusion, tous les messages sont à la fois précisément adressés et lisibles par tous, dans un cadre participatif complexe combinant la communication de masse et la communication interpersonnelle (Marcoccia à paraître).

6 Par définition, la CEMO n'est pas une communication en face à face ${ }^{3}$ L'absence de données non-verbales et paraverbales et l'absence de marqueurs personnels diminuent fortement le nombre d'indices de contextualisation des énoncés.

7 Le nombre réduit de ces indices habituellement fournis par le face à face font de la CEMO une situation de communication à faible présence sociale (Chenault 1998). Cela pose de nombreux problèmes pour la régulation des échanges, pour la gestion de leur dimension relationnelle et pour l'interprétation des énoncés.

\section{Les formes de représentation du non-verbal}

8 Afin de résoudre ces problèmes, divers procédés sont utilisés dans la CEMO pour représenter des données verbales et paraverbales. Ces procédés reposent essentiellement sur une utilisation particulière des topogrammes (Anis $1994: 86$ ).

\section{Les smileys}

9 Les smileys (ou emoticons) sont des combinaisons de topogrammes et de caractères d'imprimerie permettant de représenter de manière schématique (si on les incline à 90 degrés) des mimiques faciales comme des sourires (exemple 1), des clins d'oeil (exemple 2). des moues de colère ou de tristesse (exemple 3).

10 (Ex.l) Extrait d'un message posté au forum fr.rec.arts.bd

11 > Ce n'est pas dans la collection Mimolette, mais c'est quand même chez l'Association : « Nadia 
12 > et les autres" de l'excellent Guy Delisle. Un bouquin sans paroles, au dessin minimaliste,

13 > beau, drôle, cruel, ironique et un peu effrayant. Un délice pour psychanalyste sans doute.

14 > Ce sont des histoires de filles qui changent de peau, qui ont une sexualité de mantes

15 > religieuses, qui mangent, dévorent et muent.

16 Appollo, tu causes vachement bien de ce très beau livre, qui ne s'appelle pourtant pas « Nadia et les autres » mais « Aline et les autres ». Mais je suis d'accord avec tout, sinon, hein :-)

(Ex. 2) Extrait d'un message posté au forum fr.comp.sys.mac Bruno Blondeau wrote :

18 > Vu que Quartz semble déjà bien débuté, c'est possible. Disons que Quartz, ça fait 15 ans qu'il a déjà bien débuté :-)

(Ex. 3) Extrait d'un message posté au forum fr.comp.sys.mac

Jean Schmit a écrit :

$>>$ Dan DT wrote :

$>>>$ Qlq a une ID ?

>> Apple CD-ROM 5.3.1

Oui. je sais, mais avec cette version ou Gestionnaire CD/DVD du 8.5.1 modifié, alors c'est DirectCd qui ne va plus :-(

Par leur fonction, on peut assimiler les smileys à des pictogrammes. On peut noter que la combinaison de signes graphiques pour former un symbole figuratif de mimique faciale repose sur le même principe que la combinaison de kinèmes en kinémorphèmes dans la théorie de Birdswhistell (Cosnier \& Brossard 1984 : 10-11).

Une analyse approfondie des smileys (Marcoccia 2000) nous a permis de mettre en évidence leurs fonctions :

Un smiley peut être expressif : il sert à décrire l'état d'esprit du locuteur (la joie, comme dans l'exemple 1, la colère comme dans l'exemple 3). Il a une fonction d'autoportrait ou d'illustration de celui qui produit le message (Mourlhon-Dallies \& Colin 1995). Wilson (1993) parle de « indicators of feelings » pour désigner cette catégorie de smileys.

Le smiley peut être une aide apportée au destinataire pour qu'il puisse aisément interpréter les énoncés. On peut, comme Mourlhon-Dallies \& Colin (1995), parler de smiley interprétatif. Le smiley " clin d'œil » (exemple 2) a généralement cette fonction car il permet de lever les ambiguïtés des énoncés ironiques ou humoristiques.

Le smiley permet au locuteur d'indiquer la relation qu'il désire instaurer avec son lecteur. Utiliser un smiley "souriant » peut être ainsi à la Ibis une manière d'exprimer son émotion et de donner une tonalité particulière à l'échange.

30 Le smiley est un procédé de politesse, un moyen de désamorcer le caractère offensant d'un message (comme dans l'exemple 2).

31 On peut rapprocher ces quatre fonctions des fonctions habituelles du nonverbal et du paraverbal dans la communication en face à face. La fonction expressive du non-verbal et du paraverbal se fonde sur l'expression faciale des émotions de base (Ekman 1993). La fonction interprétative du non-verbal renvoie à la "mimogestualité connotative » (Cosnier \& Brossard, 1984 : 13-14), qui participe à la constitution et à l'interprétation 
des énoncés. Par ailleurs, la communication non-verbale et paraverbale contribue au maintien de la relation et à la synchronisation interactionnelle (Cosnier \& Brossard 1984 : 25). Enfin, le non-verbal peut être vu comme contribuant à la politesse, parce qu'il permet de limiter le travail cognitif de l'interlocuteur et de maintenir une relation satisfaisante.

\section{Les autoportraits}

La signature des messages joue aussi pleinement une fonction de marqueur personnel, de signe autocentrique (Harris 1993: 191). La signature est d'ailleurs parfois accompagnée d'une autre forme de représentation du non-verbal par l'écrit: l'autoportrait. 11 s'agit de combinaisons de topogrammes et d'alphagrammes, à la fois ludiques et expressives, représentant un portrait de celui qui envoie le message.

(Ex. 4) Extrait d'un message envoyé au forum fr.comp.sys.mac

le problème c'est que chez moi, il marche pas ce plug-in. Il plante netscape systématiquement quand il faut lire un fichier AIFF.

\section{- BEATRICE}

$\& \&\left(^{\circ} !^{\circ}\right)$

........uuu.....uuu -

Les autoportraits - comme les autres procédés de représentation du nonverbal - ont un rôle proche des marqueurs personnels (apparence physique. «look») car ils permettent de limiter l'anonymat des auteurs de messages. Le comportement et l'identité des auteurs se manifestent dans la composition de leurs textes (Harris 1993 : 180).

\section{La ponctuation expressive}

La ponctuation n'est pas seulement utilisée dans des combinaisons graphiques mais aussi dans sa valeur expressive, émotive et affective, par démultiplication du même signe :

\section{(Ex. 5) Extrait d'un message envoyé au forum fr.rec.arts.bd}

Frank Margerin dédicace ses albums. Mercredi 5 mai entre 17 h 30 et 19 h 30 au Marque Page, 195 bis rue Raymond Losserand dans le 14e arrondissement de Paris (quartier plaisance) Venez prendre un verre avec nous, même la boisson aura des bulles !!!

Le redoublement du point d'exclamation est un procédé très utilisé dans la CEMO ; il repose sur des conventions d'écriture habituellement utilisées dans la mise en scène de l'oral à l'écrit et permet de simuler des données non-verbales (mimique de surprise ou de joie) et paraverbales (intonation). 


\section{Les capitales} CEMO ont les mêmes fonctions que le non-verbal et le paraverbal dans la communication orale: fonction expressive, interprétative, marqueur de relation, procédé de politesse, marqueur personnel, fonction d'amplification.

ces procédés graphiques permettent une meilleure gestion de la relation interpersonnelle. Leur utilisation montre que les fonctions assumées par le non-verbal et le paraverbal dans la communication orale sont indispensables pour assurer une communication satisfaisante dans les groupes : ils servent à la définition contextuelle 
de la situation (le cadrage) et à l'expression des émotions, nécessaire pour la structure et le maintien des groupes (Heise \& O'Brien 1993).

On peut aussi considérer que l'utilisation de ces procédés contribue à l'émergence de la communauté, en lui associant un ensemble de conventions et de signes distinctifs (Baym 1998 : 62). Comme la Nétiquette. les procédés de représentation du non-verbal ont alors une fonction sociale car ils permettent aux utilisateurs de forums de discussion de marquer leur appartenance à une communauté virtuelle, en manifestant leur maitrise de ses codes et de ses protocoles.

Par ailleurs, on ne doit pas négliger le caractère esthétique de ces procédés graphiques, qui renvoient à la tradition du figuratif dans l'écrit.

Enfin, on peut noter qu'en réintégrant la dimension pictographique dans l'écrit, la CEMO opère un étonnant retour aux sources de l'écriture.

\section{BIBLIOGRAPHIE}

ANIS J., « Pour une graphématique des usages : le cas de la ponctuation dans le dialogue télématique ». LINX. 1994. n 31, pp. 81-97.

ANIS J., Texte et ordinateur. L'écriture réinventée ? De Boeck. Bruxelles, 1998.

BAYM N. K., "The Emergence of On-Line Community ", in S. G. Jones (éd.), Cybersociety 2.0. Revisiting Compitter-Mediated Communication and Community, Sage, Thousand Oaks. 1998. pp. 35-68.

CHENAUlT B. G., « Developing Personal and Emotional Relationships Via Computer-Mediated Communication ». Computer-Mediated Communication Magazine. mai 1998, vol. 5, n 5. Publication électronique: www.december.com/cmc/mag/1998/may/chenault.html. Colot M. \& BELMORE N., « Electronic Language: A new variety of English », in S. C. Herring (éd.), Computer-Mediated Communication. Linguistic, Social and Cross-Cultural Perspectives. John Benjamins, Amsterdam/ Philadelphia, 1996, pp. 13-28.

COSNIER J. \& BROSSARD A., « Communication non-verbale : co-texte ou contexte ? », in J. Cosnier \& A. Brossard (éds), La communication non-verbale. Delachaux et Niestlé, Neuchâtel, 1984. pp. 1-29. Ekman P., « L'expression des émotions », in B. RIME \& K. S. SCHERER (éds), Les émotions, Delachaux et Niestlé, Neuchâtel, 1993 (première éd. 1980). pp. 183-201.

FOUSER R. \& CHUNGMIN L., " The pragmatics of orality in English, Japanese and Korean computer-mediated communication ». in L. Pemberton \& S. Shurville (eds). Words on Web, Intellect Books. Exeter-Po.tland, 2000, pp. 52-62.

J. COSNIER \& C. KERBRAT-Orecchioni (éds). Décrire la conversation. Presses Universitaires de Lyon, Lyon, 1987, pp. 167-198.

HARRIS R., La sémiologie de l'écriture, CNRS éditions, Paris, 1993.

HEISE D.R. \& O'BRIEN J., « Emotion Expression in groups ». in M. Lewis \& J. M. Haviland (eds), Handbook of Emotions, Guilford Press. Londres/New York, 1993, pp. 489-497. 
MARCOCCIA M., «La Normalisation des comportements communicatifs sur Internet : étude sociopragmatique de la Nétiquette », in N. Guégen \& L. Tobin (éds). Communication, société et internet, L'Harmattan, Paris. 1998. pp. 15-32.

MARCOCCIA M., «Les Smileys : une représentation iconique des émotions dans la communication médiatisée par ordinateur ", in C. Plantin. M. Doury \& V. Traverso (éds), Les émotions dans les interactions, ARCI, Presses Universitaires de Lyon, Lyon. 2000. pp. 249-263.

MARCOCCIA M., « On-Line Polylogues : conversation structure and participation framework in Internet Newsgroups ». Journal of Pragmatics, à paraître.

MELANÇON B., Sevigne@Internet. Éditions Fides, Montréal, 1996. MOURLHON-DALLIES F. \& COLIN $\mathrm{J}-Y$. ., « Les rituels énonciatifs des réseaux informatiques entre scientifiques ». Les Carnets du CEDISCOR, n³, 1995, pp. 161-172.

PANCKHURST R., " Marques typiques et ratages en communication médiée par ordinateur ", in Actes du colloque CIDE 98 (Rabat, 15 au 15 avril 98), Luropia Productions, Paris. 1998. pp. 31-43.

SCHERER K. R., « Les fonctions des signes non verbaux dans la conversation », in J. COSNIER \& A. BROSSARD (éds), La communication non-verbale. Delachaux et Niestlé, Neuchâtel, 1984 (première éd. 1980), pp. 71-100. YATES S.J., « Oral and Written Linguistic Aspects of Computer Conferencing: A Corpus Based Study ", in S.C. HERRING (éd.), Computer-Mediated Communication. Linguistic, Social and Cross-Cultural Perspectives, John Benjamins, Amsterdam/Philadelphia. 1996, pp. 29-46.

WILSON A., « A Pragmatic device in electronic communication », Journal of Pragmatics. $n^{\circ} 19$ (4), 1993, pp. 389-392.

\section{NOTES}

1. Il s'agit de forums de discussion très différents, consacrés à la bande dessinée, au football et aux ordinateurs Macintosh : fr.rec.arts.bd, fr.rec.sports.football, fr.comp.sys.mac.

2. Pour l'anglais, voir les travaux de Colot \& Belmore (1996), Yates (1996). Pour le français, voir Anis (I99X), Panckhurst (1998). Pour le japonais et le coréen, voir Fouser, Inoue \& Lee (2000).

3. Ce n'est pas vrai pour la Communication Médiatisée par Ordinateur en général, qui peut combiner le courrier électronique a la visioconférence en joignant à un message l'image, filmée par une Webcam, de celui qui l'a écrit.

\section{AUTEUR}

\section{MICHEL MARCOCCIA}

Université de Technologie de Troyes/Tech-CICO 